\title{
HYPERLATTICE FORMED BY THE IDEMPOTENTS OF A HYPERRING
}

\author{
IㅐIII \\ A. ASOKKUMAR

\begin{abstract}
In this paper we prove that under certain conditions, the idempotent elements of a hyperring form a hyperlattice and the orthogonal idempotnet elements form a quasi-distributive hyperboolean algebra.
\end{abstract}

\section{Introduction}

The theory of hyperstructures was introduced in 1934 by Marty $[8]$ at the $8^{\text {th }}$ congress of Scandinavian Mathematicians. This theory has been subsequently developed by the contribution of various authors. Some basic definitions and propositions about the hyperstructures are found in [2] and [10]. Krasner [7] has studied the notion of hyperfields, hyperrings and then some researchers, namely, Dasic [3], Davvaz [4, 5], Gontineac [6], Massouros [9], Vougiouklis [10] and others followed him.

Hyperrings are essentially rings with approximately modified axioms in which addition is a hyperoperation and multiplication is a binary operation. Basic definitions and results about the theory of lattices and Boolean algebra are found in [1]. Throughout this paper we are using the definition of a hyperlattice as discussed by Zhao Bin, Han Sheng Wei, Xiao Ying [11].

In this paper the concepts of hyperrings and hyperlattices are discussed. Also, we relate Boolean hyperring with hyperboolean algebra.

\section{Basic definitions and notations}

This section explains some of the basic definitions that have been used in the sequel. A hyperoperation $*$ on a non-empty set $H$ is a mapping of $H \times H$ into the family of non-empty subsets of $H$ (i.e., $x * y \subseteq H$, for $x, y \in H$ ). In the sense of Marty [8], a hypergroup $(H, *)$ is a non-empty set $H$ equipped with a hypercomposition $*$, which satisfies the following axioms:

(i) $(x * y) * z=x *(y * z)$ for every $x, y, z \in H$ (the associative axiom).

Received December 21, 2005.

2000 Mathematics Subject Classification. Primary 20N20.

Key words and phrases. Canonical hypergroup, hyperring, hyperlattice, hyperboolean algebra, quasi-distributive hyperboolean algebra. 
(ii) $x * H=H * x=H$ for every $x \in H$ (the reproductive axiom).

The comprehensive review of the theory of hypergroups appears in [2]. Now we recall the following definition of a hyperring from Krasner [7].

Definition 2.1. A hyperring is an algebraic structure $(R,+, \cdot)$ which satisfies the following axioms.

(1) $(R,+)$ is an abelian canonical hypergroup under the hyperoperation + . That is,

(i) for every $x, y, z \in R, x+(y+z)=(x+y)+z$;

(ii) for every $x, y \in R, x+y=y+x$;

(iii) there exits $0 \in R$ such that $0+x=x$ for all $x \in R$;

(iv) for every $x \in R$ there exists an unique element denoted by $-x \in R$ such that $0 \in x+(-x)$

(v) for every $x, y, z \in R, z \in x+y$ implies $y \in-x+z$ and $x \in z-y$.

(2) $(R, \cdot)$ is a semigroup under the multiplication - having $0 \in R$ as a bilaterally absorbing element. That is,

(i) for every $x, y, z \in R, x \cdot(y \cdot z)=(x \cdot y) \cdot z$;

(ii) $x \cdot 0=0 \cdot x=0$ for all $x \in R$.

(3) The multiplication $\cdot$ is distributive with respect to the hyperoperation + . That is, for every $x, y, z \in R, x \cdot(y+z)=x \cdot y+x \cdot z$, and $(x+y) \cdot z=x \cdot z+y \cdot z$ hold.

We denote $x y$ instead of $x . y$. In a hyperring $R$, suppose $S, T \subseteq R$ and $x \in R$, then $S+T=\bigcup_{s \in S, t \in T} s+t, S T=\{a b \mid a \in S$ and $b \in T\}$. Moreover $x+T=\bigcup_{t \in T} x+t$ and $x T=\{x t \mid t \in T\}$ also $-T=\{-t \mid t \in T\}$.

The following elementary facts in a hyperring follow easily from the axioms.

(i) $-(-a)=a$ for every $a \in R$;

(ii) 0 is the unique element such that for every $a \in R$ there is an element $-a \in R$ with the property that $0 \in a+(-a)$;

(iii) $a+R=R$ for all $a \in R$;

(iv) $-(a+b)=-a-b$ for every $a, b \in R$;

(v) $x(-y)=-(x y)=(-x) y$ for every $x, y \in R$.

In a hyperring $R$ if there exists an element $1 \in R$ such that $1 a=a 1=a$ for every $a \in R$ then the element 1 is called the identity element of the hyperring $R$. In fact, the element 1 is unique. Further if $a b=b a$ for every $a, b \in R$ then the hyperring $R$ is called a commutative hyperring. An element $a$ of a hyperring $R$ is called idempotent if $a=a^{2}$. A hyperring $R$ is called a Boolean hyperring if every element $a$ of the hyperring $R$ is an idempotent. Any two elements $x, y$ of a hyperring $R$ are said to be orthogonal if $x y=0$ and $y x=0$.

Example 2.2. The set $R=\{0,1\}$ with the following hyperoperation + and multiplication $\cdot$ is a hyperring. 


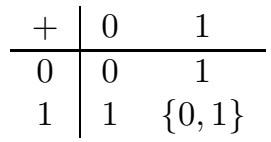

\begin{tabular}{l|ll}
$\cdot$ & 0 & 1 \\
\hline 0 & 0 & 0 \\
1 & 0 & 1
\end{tabular}

Example 2.3. M. Krasner [7] constructed a class of hyperrings as follows.

Let $(P,+, \cdot)$ be a ring and $G$ be a normal subgroup of its multiplicative semi-group (i.e., $x G=G x$ for every $x \in P$ ). Consider the set $\bar{P}=\{\bar{x}=x G \mid x \in P\}$ of classes modulo $G$.

Define hyperaddition $\oplus$ and multiplication $\otimes$ on $\bar{P}$ as follows.

For any two elements $\bar{x}, \bar{y} \in \bar{P}, \bar{x} \oplus \bar{y}=x G \oplus y G=\{(x p+y q) G \mid p, q \in G\}$ and $\bar{x} \otimes \bar{y}=x G \otimes y G=x y G$ then $\bar{P}$ becomes a hyperring.

Now we recall the definition of lattice as in [1].

Definition 2.4. A lattice $(L, \vee, \wedge)$ is a non-empty set with two binary operations $\wedge$ and $\vee$ satisfying the following properties.

(L1) for every $a \in L, a=a \vee a$ and $a=a \wedge a$,

(L2) for every $a, b \in L, a \vee b=b \vee a$ and $a \wedge b=b \wedge a$,

(L3) for every $a, b, c \in L,(a \vee b) \vee c=a \vee(b \vee c)$ and $(a \wedge b) \wedge c=a \wedge(b \wedge c)$,

(L4) for every $a, b \in L, a=a \wedge(a \vee b)$ and $a=a \vee(a \wedge b)$.

Definition 2.5.([11]) A hyperlattice $(L, \vee, \wedge)$ is a non-empty set with two hyperoperations $\wedge$ and $\vee$ such that the following properties hold.

(H1) for every $a \in L, a \in a \vee a$ and $a \in a \wedge a$,

(H2) for every $a, b \in L, a \vee b=b \vee a$ and $a \wedge b=b \wedge a$,

(H3) for every $a, b, c \in L,(a \vee b) \vee c=a \vee(b \vee c)$ and $(a \wedge b) \wedge c=a \wedge(b \wedge c)$,

(H4) for every $a, b \in L, a \in a \wedge(a \vee b)$ and $a \in a \vee(a \wedge b)$.

Example 2.6. Let $L=\{a, b\}, \vee$ and $\wedge$ be two hyperoperations defined on $L$ as follows.

\begin{tabular}{c|cc}
$\vee$ & $a$ & $b$ \\
\hline$a$ & $\{a, b\}$ & $\{a, b\}$ \\
$b$ & $\{a, b\}$ & $\{b\}$
\end{tabular}

\begin{tabular}{c|cc}
$\wedge$ & $a$ & $b$ \\
\hline$a$ & $\{a, b\}$ & $\{b\}$ \\
$b$ & $\{b\}$ & $\{b\}$
\end{tabular}

It can be easily verified that $\vee$ and $\wedge$ satisfy (H1)-(H4) and therefore $(L, \vee, \wedge)$ is a hyperlattice. For any element $x$ and any subset $S$ of a hyperlattice $L, x \vee S$ means the set $\cup\{x \vee a \mid a \in S\}$ and by $x \wedge S$ we mean the set $\cup\{x \wedge a \mid a \in S\}$.

Theorem 2.7. Let $R$ be a hyperring with identity 1 and $B$ be the set of all idempotent elements of $R$. Suppose that idempotents are central and for $x, y \in B$, every element of the set $x+y-x y$ is idempotent and $x, y \in x+y-x y$. Moreoever, if $(x+y-x y)(1-z)=$ $x+y-x y-x z-y z+x y z$ holds for every $x, y, z \in B$ then $B$ is a hyperlattice.

Proof. For $x, y \in B$, define hyperjoin $\vee$ and hypermeet $\wedge$ on $B$ as $x \vee y=x+y-x y$ and $x \wedge y=\{x y\}$. Now $x=x^{2}=x \wedge x$ and therefore we say $x \in x \wedge x$. 
We know that $0 \in x-x$, so $x+0 \subseteq x+(x-x)=x+x-x=x+x-x^{2}=x \vee x$. Thus $x \in x \vee x$. Since idempotents are central $y x=x y$. This means that $y \wedge x=x \wedge y$. Since $R$ is a hyperring we get $x+y=y+x$. Now $x \vee y=x+y-x y=y+x-y x=y \vee x$. Suppose $x, y, z \in B$ then

$$
\begin{aligned}
x \vee(y \vee z) & =x \vee(y+z-y z) \\
& =\cup\{x \vee r \mid r \in y+z-y z\} \\
& =\cup\{x+r-x r \mid r \in y+z-y z\} \\
& =\cup\{x+r(1-x) \mid r \in y+z-y z\} \\
& =x+(y+z-y z)(1-x) \\
& =x+(y+z-y z)-(y+z-y z) x \\
& =x+y+z-y z-y x-z x+y z x \\
& =x+y+z-x y-y z-z x+x y z .
\end{aligned}
$$

Also

$$
\begin{aligned}
(x \vee y) \vee z & =(x+y-x y) \vee z \\
& =\cup\{r \vee z \mid r \in x+y-x y\} \\
& =\cup\{r+z-r z \mid r \in x+y-x y\} \\
& =\cup\{z+r-r z \mid r \in x+y-x y\} \\
& =\cup\{z+r(1-z) \mid r \in x+y-x y\} \\
& =z+(x+y-x y)(1-z) \\
& =z+x+y-x y-x z-y z+x y z \\
& =x+y+z-x y-y z-z x+x y z
\end{aligned}
$$

Thus $x \vee(y \vee z)=(x \vee y) \vee z$.

Moreover

$$
\begin{aligned}
x \wedge(x \vee y) & =x \wedge(x+y-x y) \\
& =\cup\{x \wedge r \mid r \in x+y-x y\} \\
& =\cup\{x r \mid r \in x+y-x y\} \\
& =x(x+y-x y) .
\end{aligned}
$$

Since $x \in x+y-x y$ we get $x x \in x(x+y)-x x y$. That is, $x \in x(x+y)-x x y=$ $x((x+y)-x y)=x \wedge(x \vee y)$. Thus $x \in x \wedge(x \vee y)$. Now $x \vee(x \wedge y)=x \vee(x y)=$ $x+x y-x x y=x+x y-x y$. Since $0 \in x y-x y$, we have $x=x+0 \in x+x y-x y=x \vee(x \wedge y)$. Hence $x \in x \vee(x \wedge y)$. Thus $B$ is a hyperlattice.

Theorem 2.8. Let $R$ be a commutative Boolean hyperring with identity 1. Suppose that for any $x, y \in R, x, y \in x+y-x y$ and if $(x+y-x y)(1-z)=x+y-x y-x z-y z+x y z$ holds for every $x, y, z \in R$ then $R$ is a hyperlattice. 


\section{Quasi-distributive hyperboolean algebra}

In lattice theory we have a result that the two abstract systems namely, Boolean ring with identity and Boolean algebra are equivalent. In this section we try to extend the result to hyperstructures and get under certain conditions, the set of all orthogonal idempotent elements of a hyperring together with identity element, form a hyperboolean algebra.

Definition 3.1. A hyperlattice $(L, \vee, \wedge)$ is said to be a quasi-distributive hyperlattice if $a \wedge(b \vee c)=(a \wedge b) \vee(a \wedge c)$ holds for every $a, b, c \in L$.

Definition 3.2. A hyperlattice $(L, \vee, \wedge)$ is said to be a distributive hyperlattice if $a \wedge(b \vee c)=(a \wedge b) \vee(a \wedge c)$ and $a \vee(b \wedge c)=(a \vee b) \wedge(a \vee c)$ hold for every $a, b, c \in L$.

Definition 3.3. Let $(L, \vee, \wedge)$ be a hyperlattice. An element $a \in L$ is called an all element if $a \in a \vee x$ and $x \in a \wedge x$ for all $x \in L$. The set of all, all elements of $L$, is denoted by $I$.

Definition 3.4. An element $b$ in a hyperlattice $(L, \vee, \wedge)$ is called a zero element of $L$ if $x \in b \vee x$ and $b \in b \wedge x$ for all $x \in L$. The set of all zero elements of $L$ is denoted by $O$.

Definition 3.5. A hyperlattice $(L, \vee, \wedge)$ is said to have an all element (resp. a zero element) if $I$ is non-empty (resp. $O$ is non-empty).

Definition 3.6. A hyperlattice $(L, \vee, \wedge)$ is said to be complemented if for every $a \in L$ there exists elements $a^{\prime} \in L, a_{i} \in I$ and $a_{0} \in O$ such that $a_{i} \in a \vee a^{\prime}$ and $a_{0} \in a \wedge a^{\prime}$.

Definition 3.7. A hyperlattice $(L, \vee, \wedge)$ with $O, I$ is said to be a hyperboolean algebra if $L$ is distributive and complemented. Instead of the two distributive laws if for every $a, b, c \in L$ only one distributive law, namely $a \wedge(b \vee c)=(a \wedge b) \vee(a \wedge c)$ holds then $(L, \vee, \wedge)$ is called a quasi-distributive hyperboolean algebra.

Theorem 3.8. Let $R$ be a hyperring with identity $1 \neq 0$ and $A$ be the set of all orthogonal idempotent elements of $R$. Suppose that for any two elements $x, y$ of $A$, $x, y \in x+y-x y \subseteq A$. Moreover, if $1-a \subseteq A \cup\{1\}$ for every $a \in A \cup\{1\}$ and $(x+y-x y)(1-z)=x+y-x y-x z-y z+x y z$ holds for every $x, y, z \in A \cup\{1\}$ then $A \cup\{1\}$ is a quasi-distributive hyperboolean algebra.

Proof. If $1 \in A$ then for any $1 \neq x \in A$ we get $1 x=0$. This implies that $x=0$. That is, $A=\{0,1\}$. By the hypothesis $0,1 \in 0+1-0=\{1\}$. This means that $0=1$, which is a contradiction. Thus identity element 1 is not an element of $A$.

Let $A^{\prime}=A \cup\{1\}$. Define hyperjoin $\vee$ and hypermeet $\wedge$ on $A^{\prime}$ as follows. For every $x, y \in A^{\prime}, x \vee y=x+y-x y$ and $x \wedge y=\{x y\}$. It is clear that $A^{\prime}$ is a hyperlattice.

Suppose that $a, b, c \in A^{\prime}$. If $a=b=c$ then $a \wedge(b \vee c)=a \wedge(a \vee a)=a a+a a-a a a=$ $a+a-a$ and $(a \wedge b) \vee(a \wedge c)=a \vee a=a+a-a$. Thus $a \wedge(b \vee c)=(a \wedge b) \vee(a \wedge c)$ 
If $a=b$ and $c \neq a, c \neq b$ then $a \wedge(b \vee c)=a a+a c-a a c=a+a c-a c$ and $(a \wedge b) \vee(a \wedge c)=a a+a c-a a c=a+a c-a c$. Thus $a \wedge(b \vee c)=(a \wedge b) \vee(a \wedge c)$.

If $a=c$ and $b \neq a, b \neq c$ then $a \wedge(b \vee c)=a \wedge(b+a-b a)=a b+a-a b a$ and $(a \wedge b) \vee(a \wedge c)=a b \vee(a \wedge a)=a b \vee a=a b+a-a b a$. Therefore, we see $a \wedge(b \vee c)=(a \wedge b) \vee(a \wedge c)$

Now for the case when $b=c$ and $a \neq b, a \neq c$, we get $a \wedge(b \vee c)=a \wedge(b \vee b)=a b+a b-a b$ and $(a \wedge b) \vee(a \wedge c)=a b \vee a b=a b+a b-a b a b$. If $a, b, c \in A$ then $a \wedge(b \vee c)=0=$ $(a \wedge b) \vee(a \wedge c)$. If $b=c \in A$ and $a=1$ then $a \wedge(b \vee c)=b+b-b=(a \wedge b) \vee(a \wedge c)$. If $a \in A$ and $b=c=1$ then $a \wedge(b \vee c)=a+a-a=(a \wedge b) \vee(a \wedge c)$. Thus $a \wedge(b \vee c)=(a \wedge b) \vee(a \wedge c)$.

We suppose now $a \neq b, a \neq c, b \neq c$.

If $a, b, c \in A$ then $a \wedge(b \vee c)=0=(a \wedge b) \vee(a \wedge c)$.

If $b, c \in A$ and $a=1$ then $a \wedge(b \vee c)=1 \wedge(b+c-b c)=b+c$ and $(a \wedge b) \vee(a \wedge c)=$ $b \vee c=b+c-b c=b+c$.

If $a, c \in A$ and $b=1$ then $a \wedge(b \vee c)=a \wedge(1+c-c)=a+a c-a c=a$ and $(a \wedge b) \vee(a \wedge c)=a \vee(a c)=a \vee 0=a$. In the same way, for $a, b \in A$ and $c=1$, we obtain that $a \wedge(b \vee c)=(a \wedge b) \vee(a \wedge c)$.

Thus $a \wedge(b \vee c)=(a \wedge b) \vee(a \wedge c)$ is true for every $a, b, c \in A^{\prime}$ and hence $A^{\prime}$ is a quasi-distributive lattice.

For any element $a \in A^{\prime}, 1+0 \in 1+a-a=a+1-a 1=a \vee 1$. That is, $1 \in a \vee 1$. Since $a 1=a$, we see that $a \in 1 \wedge a$. Thus the identity element of the hyperring is an all element of the hyperlattice $A^{\prime}$. Clearly, $x \in 0 \vee x$ and $0 \in 0 \wedge x$ for all $x \in A^{\prime}$. Thus the zero element of the hyperring is a zero element of the hyperlattice $A^{\prime}$. Let $0 \neq a \in A^{\prime}$, then $0 \in a-a=a-a^{2}=a(1-a)$. This implies that $0=a a^{\prime}$ for some $a^{\prime} \in 1-a \subseteq A^{\prime}$. That is, $0 \in a \wedge a^{\prime}$. Since $a^{\prime} \in 1-a$ we see that $1 \in a^{\prime}+a=a^{\prime}+a-0=a^{\prime}+a-a^{\prime} a=a \vee a^{\prime}$. In fact every element of $1-a$ is a complement of $a$. Hence $A^{\prime}$ is a complemented quasidistributive lattice with all elements set $I$ and zero elements set $O$. That is, $A^{\prime}$ is a quasi-distributive hyperboolean algebra.

\section{Acknowledgement}

I would like to thank the referee of this paper for the valuable suggestions.

\section{References}

[1] G. Birkhoff, Lattice Theory, American Mathematical Society Colloguium Publications, Province, Rhode Island, 1940.

[2] P. Corsini, Prolegomena of Hypergroup Theory, Second edition, Aviani editor, 1993.

[3] V. Dasic, Hypernear-rings, Proc. Fourth Int. Congress on Algebraic Hyperstructures and Applications(AHA 1990), World Scientific, (1991), 75-85.

[4] B. Davvaz, H $\nu$-near-rings, Math. Japonica, 52(2000), 387-392.

[5] B. Davvaz and A. Koushky, On hyperring of polynomials, Italian Journal of Pure and Applied Mathematics-N. 15(2004), 205-214. 
[6] V. M. Gontineac, On hypernear-rings and H-hypergroups, Proc. Fifth Int. Congress on AHA., Jasi Rumania, Hadronic Press, Inc. (1993), 171-179.

[7] M. Krasner, A class of hyperrings and hyperfields, Int. J. Math. and Math. Sci. 2(1983), 307-312.

[8] F. Marty, Sur une generalization de la notion de groupe, $8^{\text {th }}$ Congress Math. Scandenaves, Stockholm, (1934), 45-49.

[9] G. G. Massouros, The hyperringoid, Multi. Val. Logic. 3(1998), 217-234.

[10] T. Vougiouklis, Hyperstructures and Their Representations, Hadronic Press, Inc., 115, Palm Harber, USA, 1994.

[11] Zhao Bin, Han Sheng Wei and Xiao Ying, The ideal on hyperlattices, http:\www.paper.edu.cn, uploading date 25 th April 2005.

Department of Mathematics, Aditanar College of Arts and Science, Tiruchendur-628216, Tamilnadu, India.

E-mail: ashok_a58@yahoo.co.in 